\title{
NEONATAL SCIATIC PALSY FROM INJECTION INTO THE UMBILICAL CORD
}

\author{
N. E. Shaw, London, England \\ From the Children's Hospital, Sheffield
}

Paralysis and gangrene of the lower limb in the newborn have been reported as occurring together or independently. Ehrenfest (1931) reported several of Bernhardt's cases of sciatic paralysis after extraction by the foot. He stated: "Bruns expressed the opinion that circumscribed paralyses of the lower extremity of newborn infants are probably not uncommon, but are overlooked or not reported, because they are likely to disappear promptly."

Michaëlis (1931) reported complete lateral popliteal paralysis of an infant of six weeks. The pregnancy and delivery had been normal, and the etiology was not discovered. Meyer (1931) described three children with neonatal paralysis of the lower extremity: one attributed to traction on the limb during breech delivery, one associated with fracture of the femoral shaft, and another complicating purulent meningitis that ultimately proved fatal.

Lateral popliteal paralysis in the right leg of a child delivered by Caesarean section was described by Lust and Berrewaerts (1932). The palsy recovered in twenty days and the cause was ascribed to "faulty intra-uterine position with compression." Mills (1949) described eight cases of neonatal sciatic palsy and referred to one other which he had traced. The etiology remained unproven, but it was suspected that the lesion might have been caused by injection of drugs into an umbilical artery. Bates and Page (1949) reported another child in whom nikethamide had been injected into the cord. Fahrni (1950) presented eleven examples of sciatic nerve paralysis; he suggested that direct intra-uterine pressure played a part in the etiology. Hudson, McCandless and O'Malley (1950) reported twenty cases including the eleven already reported by Fahrni.

The present paper reports three cases of neonatal sciatic palsy, two in a pair of twins and the third also in a twin. A review of the literature has failed to reveal another case in twins.

\section{CASE REPORTS}

Case 1-N. G. was admitted on July 5, 1957. He was the first-born of twin boys delivered by Caesarean section five hours before admission. Because of white asphyxia at birth, nikethamide ( 1 millilitre) had been injected into the umbilical cord. Soon afterwards a pinkish mottled discoloration developed in the skin of the left thigh and left iliac fossa. A left foot-drop was also observed.

On examination the weight was $5 \mathrm{lb} .10 \mathrm{oz}$. (2.55 kilograms), temperature 101 degrees Fahrenheit (38.3 degrees Centigrade), and pulse 166. The pinkish mottling was present on the skin of the left iliac fossa, the femoral triangle, the left popliteal fossa, and the left half of the scrotum (Fig. 1). The left foot was flaccid. The femoral pulse was palpable on both sides, but was weaker on the left. The left dorsalis pedis pulse was not palpable. The rest of the affected limb was warm and of the same colour as the right leg, and active movement at the left knee was present. A knee jerk was obtained, but the ankle jerk was equivocal. The cardiac and respiratory systems appeared normal.

Diagnosis-The signs were ascribed to spasm in the branches of the external iliac and femoral arteries from retrograde injection of nikethamide into the umbilical artery, with involvement of the parietal branch of the internal iliac artery to the sciatic nerve.

Treatment-Papaverine hydrochloride ( 5 milligrams) was given by mouth every six hours for twenty-four hours. Penicillin (250,000 units) was given intramuscularly six-hourly and streptomycin (150 milligrams) twice daily. 
Progress - Three days after admission the baby vomited bile-stained fluid. Bright red blood and mucus were seen in a small amount of faeces. The left femoral pulse was impalpable. The discoloration was spreading to the left hypochondrium. Similar discoloration about one inch in diameter appeared in the skin of the right iliac fossa. This suggested a spreading thrombophlebitis. Intravenous fluid $(4.3$ per cent dextrose with 0.18 per cent sodium chloride) containing heparin $(2,600$ units-10 milligrams) was given at a rate of 275 millilitres daily. On the next day the baby developed ileus and his condition deteriorated. Intravenous fluid was continued and gastric aspiration commenced. Heparin was discontinued. After a further two days the infant was taking fluids by mouth and passing faeces. The skin over the left iliac

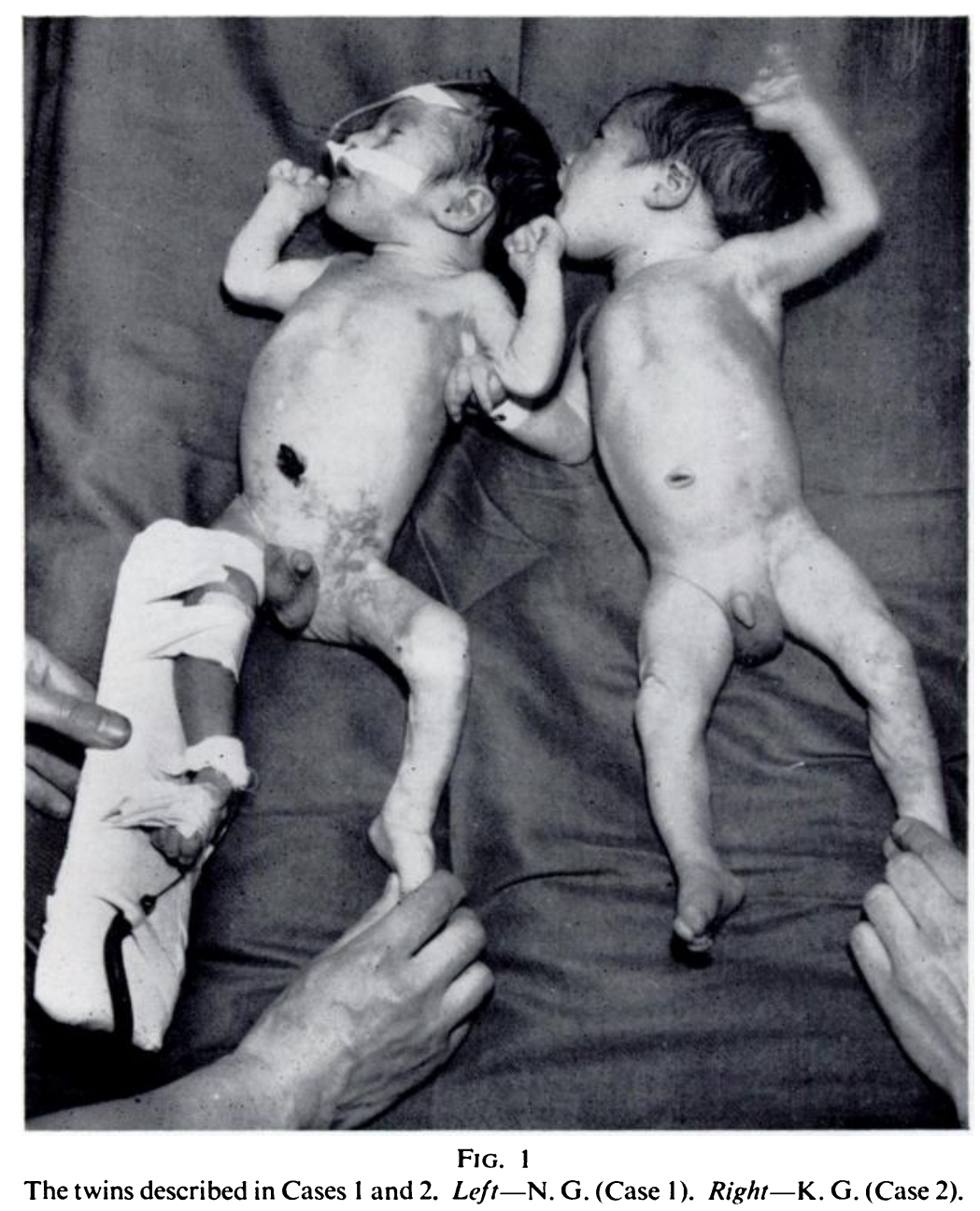

fossa became hard and there was a suggestion of developing flexion contracture of the left hip and knee. Passive exercises and splinting were instituted. A week later he was fully breast fed and gaining weight $(5 \mathrm{lb} .5 \mathrm{oz} .=2.01$ kilograms). He was discharged home.

On July 28 , twenty-three days after his first admission, the infant was readmitted with a paracolic abscess pointing in the left iliac fossa. This was incised and drained. A faecal fistula developed, but the child's progress was satisfactory, and subsequently the fistula was closed. The foot-drop has persisted.

Case 2-K. G., the younger twin-brother of N. G. (Case 1) was admitted to the Children's Hospital on July 10, 1957. Although flaccidity of the left foot was reported on July 6 it was 
two days later before he developed the pink cutaneous mottling like that seen in his brother. $\mathrm{He}$ had received an injection of nikethamide into the cord at birth.

On examination the birth weight was $5 \mathrm{lb} .11 \mathrm{oz}$. ( $2 \cdot 8$ kilograms), temperature 97.6 degrees Fahrenheit (36.4 degrees Centigrade), pulse 140, regular and the volume full.

Pink mottling was seen over small areas in the left iliac fossa and the upper quadrant of the femoral triangle, and over the medial aspect of the left knee and calf. All pulses were palpable. Foot-drop was present on the left. The skin mottling faded and no further signs developed. Treatment-Passive movements of the left

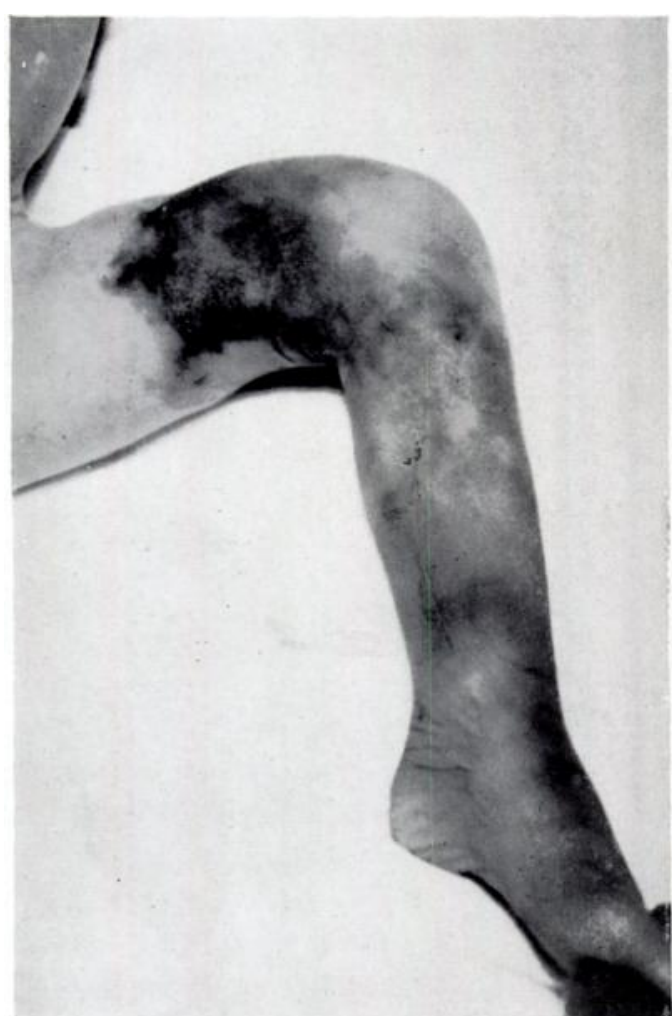

FIG. 2

Case 3. lower limb were carried out. Active movement of the limb returned in five days and the baby was discharged from hospital on July 18 , eight days after his admission.

Case 3-J. H. was admitted on January 15, 1957, three days after birth by Caesarean section after the normal delivery of a monster and the finding of another leg in the vagina. The child was suffering from white asphyxia at birth, and received an injection of nikethamide (1 millilitre) into the cord. Soon afterwards the left leg was white, and twentyfour hours later bruising was noticed in the mid-thigh (Fig. 2). On examination the left leg was cold and showed purplish mottling below the knee. The pulses of the limb were impalpable. The limb was flaccid. The haemoglobin was 6.5 grammes per cent.

Treatment-A blood transfusion of 52 millilitres was given. On January 18, 1957, left lumbar sympathectomy was performed, but the circulation did not improve. The limb became gangrenous and was amputated through the mid-thigh three days later. On February 25 the amputation stump was dry and clean. The haemoglobin content was $12 \cdot 6$ grammes per cent, red blood corpuscles $4 \cdot 3$ millions per cubic millimetre, white blood cells 17,500 per cubic millimetre, and sedimentation rate 44 millimetres in the first hour (Wintrobe). Platelets were 9,000 per cubic millimetre. A week later the child became pale and dyspnoeic, but responded to oxygen. Similar episodes occurred for a day or two, and the child died in an attack on March 8, 1957.

Necropsy-The cause of death was pulmonary oedema and inhalation of vomit. There was thrombosis of the left inferior epigastric artery which may have been related to the primary arterial defect in the left leg. No gross anatomical deformity was present.

\section{DISCUSSION}

These three cases illustrate the characteristics described in the literature.

Clinical picture-Most of the pregnancies described in the literature have been normal. There has been no common factor in foetal presentation or delivery, but in all cases there has been respiratory distress and the administration of an analeptic into the cord.

The condition may be seen immediately, but in two children six days elapsed before the palsy was noticed. The time of onset of the condition is difficult to assess because the mode 
of presentation varies according to whether the first lesion to be observed is the cutaneous manifestation or the paralysis.

Cutaneous lesions-Discrete areas of purple mottled discoloration appear over the affected parts of the limb, buttock or abdominal wall. The area of mottling is outlined by a slightly deeper purple margin, which becomes indurated and may ulcerate. The remainder of the skin of the limb may be pale, cold, and anaesthetic in the sciatic distribution.

The skin lesion may resolve, or a variable area of skin may die and slough, leaving an ulcer which is slow to heal. In one of Fahrni's cases healing took two months.

Circulatory changes-Arterial pulsation may be absent from the pale, cold limb, but it nearly always returns later. Sometimes the arterial supply is so poor that gangrene occurs, as in the third case of the present series and in one of Mills's cases.

Peripheral nerve lesion-This was recorded in all but two of thirty-two cases. It was observed immediately after birth or several hours or days later. The right leg was involved in eleven cases and the left in twenty-one. The nerve involvement varies from a mild paresis to complete flaccidity, with loss of reflexes and sensation. The lateral popliteal nerve is commonly involved, with consequent foot-drop, and this was present in our series. The skin lesion does not invariably accompany even the most severe nerve lesion.

Intra-abdominal lesions - In one of the cases reported by Hudson, McCandless and O'Malley (1950) the inferior mesenteric vessels were thrombosed and the child died from peritonitis; necropsy revealed thrombosis of the right common iliac and inferior mesenteric vessels which had caused gangrene and perforation of the pelvic colon. This knowledge enabled us to expect thrombosis of the inferior mesenteric and internal iliac group of vessels supplying the pelvic colon, rectum and bladder. Watch was kept for blood-stained stools and ileus from peritonitis. This occurred in one of my cases, but the child survived in spite of the development of a paracolic abscess.

Prognosis-Neonatal sciatic palsy from injection into the umbilical cord is a serious condition. In this series only one child recovered completely, one has some residual paralysis requiring further orthopaedic care, and one died.

Of the patients reported by Mills (1949), one infant died from cerebral haemorrhage and one from peritonitis; one infant had residual paralysis of the anterior tibial muscles. The remaining five recovered completely.

In only three of the ten survivors in Fahrni's (1950) series was recovery complete. In the other seven limbs no useful recovery was obtained in the affected muscles in spite of many months of treatment.

Occasionally paralysis of the affected limb may recover promptly and completely, but a complete or partial sciatic paralysis often remains leaving disability resembling that from poliomyelitis with concomitant failure of limb growth. Hudson, McCandless and O'Malley (1950) pointed out that the most apparent and persistent feature in all the babies is foot-drop. There may be a gradual recovery during the first six to nine months, and in a few instances recovery is complete, but in most some foot-drop persists and growth of the foot and leg is retarded. At least half an inch of leg shortening and a small foot with impaired sensation may be expected. Some of these infants require orthopaedic care to correct equinus deformity and inequality of limb growth.

The severity of the abdominal complication depends on the extent of the thrombosis of the inferior mesenteric artery. One of Mills's patients died from peritonitis after necrosis and perforation of the pelvic colon, whereas in the patient reported here the child survived although a faecal fistula developed.

Pathology-McFarland (1950) commented that the common features in Fahrni's cases were foetal shock and the injection of an analeptic into the umbilical cord. He postulated that the injection of an analeptic into the umbilical artery instead of the vein could cause spasm or thrombosis of the hypogastric artery, and the internal and external iliac vessels and their 
branches. In neonatal shock the umbilical vein is collapsed, empty and invisible, whereas the two arteries are more readily discernible. The structures supplied by the first large branch of the internal iliac artery include part of the sciatic nerve, and the muscles and the skin over the lower gluteal region. These are all involved in the syndrome.

The skin of the iliac fossa may be affected by spread of the injection into the external iliac artery and the inferior and superficial epigastric vessels, and the scrotal or vulval skin through the superficial external pudendal trunks from femoral arterial involvement. The blood supply to the pelvic colon may be jeopardised by arterial spasm or thrombosis of the inferior mesenteric artery because of retrograde spread of the injected fluid.

Mills suggested that the etiology depended on three factors: 1) the volume of fluid injected; 2) the vigour of the " milking" process, which determines how much of the fluid enters the foetal hypogastric arteries; 3) the infant's systolic blood pressure, which determines how far the retrograde injection spreads before being swept peripherally.

There may be initial arterial spasm with subsequent thrombosis simulating that after intra-arterial injection of anaesthetic solutions. Attempts have been made to reproduce the lesion in newborn kittens, and some produced a limp but the lesion itself was not demonstrated.

In the cases reported here the drug used was nikethamide, as in the cases of Mills, and Bates and Page. Cycliton was the suggested offender in the series of Hudson, McCandless and O'Malley, who pointed out that the same effects have not been seen with lobeline.

Cohen (1948) reported that intra-arterial injections of thiopentone caused mottled bluish cutaneous patches, some of which merely blistered the skin whereas others sequestrated it, leaving sloughing ulcers. He suggested that the ill effects of intra-arterial thiopentone might be due to its alkalinity (p.H. 10.4-10.6). Cycliton (p.H. 4.92) and nikethamide (p.H. 5.77) are both acid in reaction. It is unlikely that acidity plays a significant part in these lesions, because no ill effects have followed the use of lobeline (p.H. 4.8) as an analeptic. Lobeline is an alkaloid whereas cycliton and nikethamide are synthetic. Nikethamide causes vasoconstriction by local action on the vessels, a property not found when Curtis and Wright (1926) investigated lobeline.

Both cycliton and nikethamide are used in 25 per cent solution. Although the compounds themselves may be harmless enough pharmacologically, solutions of such concentration might possibly irritate the intima and so cause arterial spasm and thrombosis. Intimal injury is the precipitating factor in thrombosis (Annotation, British Medical Journal 1950).

Nerves have a regional and a longitudinal blood supply; but, in the case of the sciatic nerve, Adams (1942) reported convincing evidence for the dominance of the regional over the longitudinal blood supply. In one infant in which the sciatic nerve was sectioned after death from enteritis, there were recent haemorrhages into the nerve fascicles, while distal to it the circumference of the fascicles was waxy, suggesting shrinkage.

Holmes, Highet and Seddon (1944) pointed out that there is no effective treatment for established ischaemic nerve degeneration. If the ischaemia is transient, causing Wallerian degeneration and only slight endoneural fibrosis, then spontaneous recovery may be expected. Temporary ischaemia, presumably due to vasospasm, would account for the gradual return of function in many of the babies.

Arterial spasm in the small sciatic artery followed by thrombosis would greatly reduce the blood supply to the sciatic nerve and the buttocks, with consequent paralysis and gangrene. Treatment-It appears from the evidence available that injection of the analeptic into the umbilical artery is the cause of neonatal sciatic palsy. This is preventable.

Arterial spasm may be overcome by antispasmodic drugs such as papaverine. The action of intra-arterial procaine is evanescent. Oral papaverine, alcohol and aspirin have been used in cases attributed to thiopentone with no dramatic improvement. In our case papaverine was of doubtful value, but it was only given orally. 
Anticoagulant therapy is desirable. Heparin may be used to overcome early thrombosis. Intermittent administration of heparin is inadequate for threatened arterial thrombosis, and heparin is best given in a saline drip (Arterial Injuries, Medical Research Council 1944). Kiesewetter and Shumacker (1948) confirmed that a constantly prolonged coagulation time is more desirable and better than a fluctuating one, particularly in relation to clotting in the smaller arteries.

In the prevention of equinus and scar contraction it is necessary to use carefully padded splints because of sensory loss. Dressings to ulcerated areas are also necessary. Orthopaedic supervision is needed at least until the end of growth, and stabilisation of the foot may be performed if necessary.

\section{SUMMARY}

1. Three new cases of neonatal sciatic palsy are reported in twins. They received identical treatment after birth; in the case of the first two for identical difficulties occurring after delivery. In twins there is a greater liability to neonatal shock and so a greater incidence of umbilical injections.

2. The clinical evidence and the necropsy findings in Case 3 support the hypothesis that intra-arterial thrombosis is caused by the injection of an analeptic into the umbilical artery.

3. The clinical picture is described, with a discussion on the pathology, treatment and the reason for recovery in some cases.

I am grateful to Dr C. C. Harvey and Mr R. B. Zachary for their help, encouragement and permission to publish these cases. I am also indebted to $\mathrm{Mr} \mathrm{H}$. Jackson Burrows for his helpful criticism of this paper.

\section{REFERENCES}

Adams, W. E. (1942): The Blood Supply of Nerves. Journal of Anatomy, 76, 323.

AnNotation. Neonatal Asphyxia (1953): British Medical Journal, ii, 328.

annotation. Resuscitation of the Newborn-a Possible Danger (1950): British Medical Journal, i, 237. Arterial Injuries (1944): Medical Research Council War Memorandum No. 13. London: His Majesty's Stationery Office.

Bates, C., and Page, A. P. M. (1949): A New Neonatal Syndrome. British Medical Journal, ii, 756.

Cohen, S. M. (1948): Accidental Intra-arterial Injection of Drugs. Lancet, ii, 361, 409.

CURTIS, F. R., and Wright, S. (1926): Observations on the Action of Lobeline. Lancet, ii, 1,255.

Ehrenfest, H. (1931): Birth Injuries of the Child. Second edition. New York and London: D. Appleton \& Company.

Fahrni, W. H. (1950): Neonatal Sciatic Palsy. Journal of Bone and Joint Surgery, 32-B, 42.

Holmes, W., Highet, W. B., and Seddon, H. J. (1944): Ischaemic Nerve Lesions Occurring in Volkmann's Contracture. British Journal of Surgery, 32, 259.

Hudson, F. P., McCandless, A., and O'Malley, A. G. (1950): Sciatic Paralysis in Newborn Infants. British Medical Journal, i, 223.

Kiesewetter, W. B., and Shumacker, H. B., Jun. (1948): An Experimental Study of the Comparative Efficacy of Heparin and Dicumarol in the Prevention of Arterial and Venous Thrombosis. Surgery, Gynecology and Obstetrics, 86, 687.

Lust, M., and BerrewaerTs, M. L. (1932): Un cas de paralysie obstétricale du membre inférieur droit chez un enfant né par césarienne. Bruxelles-Médical, 12, 507.

McFarland, B. (1950): Comment on Neonatal Sciatic Palsy (Fahrni). Journal of Bone and Joint Surgery, 32-B, 47.

Meyer, M. (1931): Paralysie obstétricale des membres inférieurs. Revue d'Orthopédie, 18, 767.

MiChä̈LIS, L. (1931): Úber Peroneuslahmung beim Neugeborenen. Bruns' Beiträge zur klinischen Chirurgie, $152,629$.

Mills, W. G. (1949): A New Neonatal Syndrome. British Medical Journal, ii, 464. 